\title{
Resonance-coupling effect on broad band gap formation in locally resonant sonic metamaterials
}

\author{
Meng Chen ${ }^{\mathrm{a}}$, Dan Meng ${ }^{\mathrm{a}, \mathrm{b}}$, Han Zhang ${ }^{\mathrm{c}}$, Heng Jiang ${ }^{\mathrm{a}, \mathrm{b}}$, Yuren Wang ${ }^{\mathrm{a}, *}$ \\ a Key Laboratory of Microgravity, Institute of Mechanics, Chinese Academy of Sciences, Beijing 100190, China \\ b State Key Laboratory of Explosion Science and Technology, Beijing Institute of Technology, Beijing 100081, China \\ c State Key Laboratory of Acoustics, Institute of Acoustics, Chinese Academy of Sciences, Beijing 100190, China
}

\section{H I G H L I G H T S}

- We develop a new method of broadening the resonant band gap on the basis of coupled resonance.

- Our calculations suggest that stacked-structure LRSMs have wider band gaps.

- The bandwidth has a close relationship with the strength of coupling between resonances.

\section{A R T I C L E I N F O}

\section{Article history:}

Received 12 August 2015

Received in revised form 3 February 2016

Accepted 5 February 2016

Available online 16 February 2016

\section{Keywords:}

Locally resonant sonic metamaterials

Stacked structure

Band gap

Resonance-coupling effect

Finite element methods

\begin{abstract}
A B S T R A C T
To broaden the resonant band gap in locally resonant sonic metamaterials (LRSMs), coupled resonance was introduced through a stacked structure. Stacked-structure LRSMs are formed by orthogonally stacking square coated-steel rods and embedding them layer by layer in an epoxy matrix. Calculations suggest that stacked-structure LRSMs have wider band gaps, which are adaptable to all types of vibration polarizations. Using vibration modes and a mass-spring model, strong coupling was confirmed between the orthogonal resonances at the upper edge of the band gap, providing a wider bandwidth. Moreover, the effects of the rod width, thickness of coating material and viscoelastic damping on the band gap were investigated. The close relationship of the bandwidth with the strength of coupling between resonances was thereby demonstrated.
\end{abstract}

(C) 2016 Elsevier B.V. All rights reserved.

\section{Introduction}

Locally resonant sonic metamaterials (LRSMs) have attracted much attention for their ability to break through the mass density law and exhibit novel physical properties, such as a negative modulus, negative density and band gaps [1-8]. Typical LRSMs are made of distributed inclusions embedded in a hard matrix and have superiority in shielding low-frequency sound because of their sub-wavelength band gaps [9-13]. A key issue for the industrial application of an LRSM is the narrow bandwidth produced by the formation mechanism of the resonant band gap [14]. It has been found that relatively wide band gaps can be obtained by changing the LRSM structure [15-18] or by varying the elastic characteristics of the component materials [19-21]. However, these methods are usually based on changing intrinsic local resonance properties and thus usually limited and ineffective. Although the broadband effect of locally resonant sonic shields has been realized by combining resonances of different frequency, the manufacture of the shields is too complicated to be practical [14,19,22]. A novel LRSM is reported in this paper with the aim to use coupling resonances to broaden the band gap.

\footnotetext{
* Corresponding author.

E-mail addresses: hengjiang@imech.ac.cn (H. Jiang), yurenwang@imech.ac.cn (Y. Wang).
} 
a

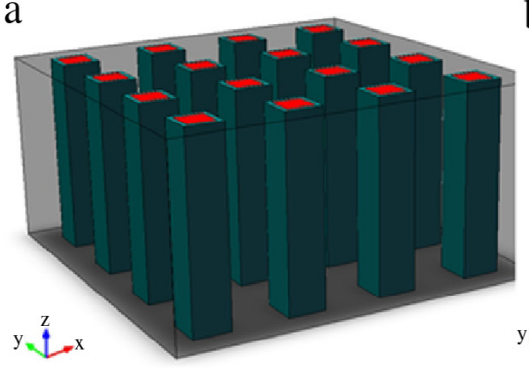

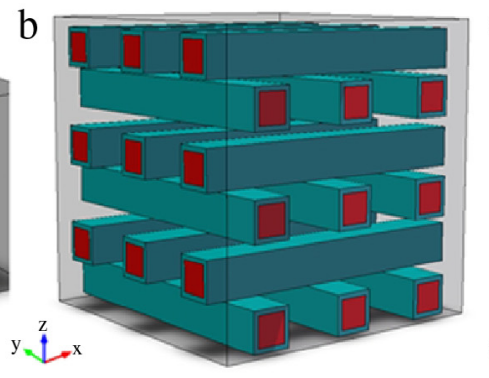

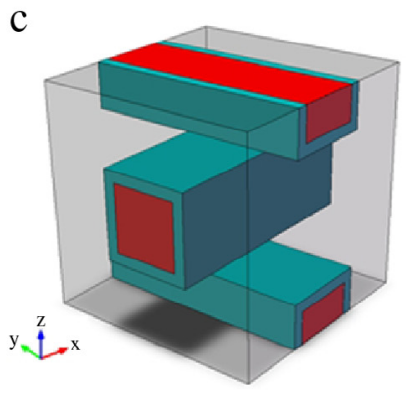

Fig. 1. Schematics of LRSMs: (a) a two-dimensional LRSM, (b) a stacked-structure LRSM, and (c) a unit cell of a woodpile structure with a simple cubic lattice.

A natural idea has been proposed to broaden the bandwidth of LRSMs using the coupling effect of resonators. The coupling of resonators produces a new vibration mode that affects and widens the band structure [23-27]. However, in the case of LRSMs having traditional structure, such as two-dimensional LRSMs with rod dispersal $[9,28-30]$ and three-dimensional LRSMs with sphere dispersal $[1,10]$, the coupling effect between resonators has been rarely reported. In traditionally structured LRSMs, the unit cell usually contains one resonator, and the formation of the band gap depends on the vibrations of the isolated resonators. The vibration mode of the resonators at the lower edge of the band gap is the vibration of the scatterers considered as particles [1,31]. In contrast, the vibration mode of the resonators at the upper edge of the band gap is the relative vibration between the matrix and scatterers [1,31]. There is only a phase difference and no correlation between the resonators in adjacent lattices. Thus, the movements of resonators in traditionally structured LRSMs are usually isolated and coupling is too weak to be observed. It is thus recognized that the introduction of complex lattices that contain multiple resonators in one unit cell is needed for LRSMs to have strong coupling between resonators.

The present paper proposes a novel LRSM with complex lattices: a structure that is stacked layer-by-layer and having orthogonal resonations [32]. Calculations suggest that there is strong coupling between the orthogonal resonations in the unit cell and that coupling produces a wider band gap for LRSMs with a stacked structure. Compared with the twodimensional LRSM having the same components, band gaps of stacked-structure LRSMs adapt to all types of vibration polarization (i.e., longitudinal and transverse) and are wider by $128 \mathrm{~Hz}$. In addition, other factors, including how the rod width, thickness of the coating material and viscoelastic damping of materials affect the bandwidth and coupling strength, were investigated.

\section{Model and method of calculation}

The structure of a stacked LRSM is shown in Fig. 1(b) and its two-dimensional LRSM variant in Fig. 1(a). The stacked structure can be obtained when the coated square column of the two-dimensional LRSM in the interval position is rotated 90 degrees. In the present study, the scatters are made of steel and the coating material is silicone rubber. Coated steel square columns were embedded in a matrix made of epoxy and the coupling effect was investigated by calculating the band gap structure and vibration modes.

Finite element methods (FEMs) are commonly used to calculate the band structure and vibration modes of LRSMs [33,34]. A distinct advantage of the FEM is the flexibility of modeling various materials with complex structure, good convergence and high precision [34]. The FEM software COMSOL Multiphysics was used to calculate the band structure and transmission. With an FEM based on the Bloch theorem, calculations of the dispersion relationship were made. The governing equation of elastic wave propagation in solids is given by

$$
\rho \frac{\partial^{2} u_{i}}{\partial t^{2}}=\sum_{j=1}^{3} \frac{\partial}{\partial x_{j}}\left(\sum_{l=1}^{3} \sum_{k=1}^{3} c_{i j k l} \frac{\partial u_{k}}{\partial x_{l}}\right), \quad(i=1,2,3),
$$

where $\rho$ is the density, $u_{i}$ is the displacement, $t$ is time, $C_{i j k l}$ denotes elastic constants, and $x_{j}$ denotes the coordinate variables $x, y$ and $z$. Here, it is assumed that the displacement varies harmonically over time. Thus, the discrete form of eigenvalue equations of the FEM in the unit cell can be written as

$$
\left(K-\omega^{2} M\right) u=0
$$

where $K$ and $M$ are respectively the stiffness and mass matrices, $u$ is the displacement and $\omega$ is the angular frequency. According to the Bloch theorem, only one unit cell needs to be considered in the calculation.

The stacked structure can be considered a simple cubic lattice. A schematic of a stacked-structure unit cell with a simple cubic lattice is shown in Fig. 1(c). The structure is assumed to be infinite and periodic in the $x, y$ and $z$ directions. Because the calculation is compressed in one unit cell, the band structure is calculated by applying the Bloch-Floquet periodic boundary 

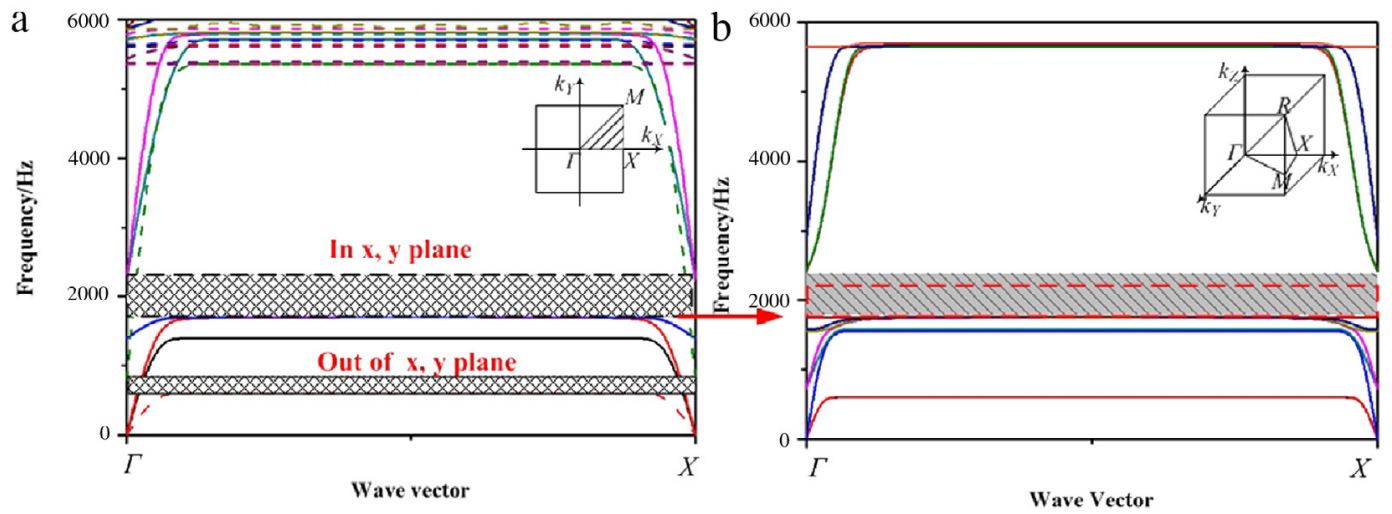

Fig. 2. Band structure of the two-dimensional LRSM (a) and stacked structure (b).

conditions for the boundaries of the unit cell along the $x, y$ and $z$ directions [35]:

$$
\begin{aligned}
& u(x+a, y, z)=u(x, y, z) e^{i\left(k_{x} \cdot a\right)}, \\
& u(x, y+a, z)=u(x, y, z) e^{i\left(k_{y} \cdot a\right)}, \\
& u(x, y, z+a)=u(x, y, z) e^{i\left(k_{z} \cdot a\right)},
\end{aligned}
$$

where $k_{x}, k_{y}$ and $k_{z}$ are the components of the Bloch wave vector in the $x, y$ and $z$ directions respectively, and $a$ is the lattice constant. With a given wave number $k$, a series of eigenfrequencies and corresponding eigenmodes can be obtained employing the FEM. The band structure can be obtained by sweeping $k$ along the boundaries of the first Brillouin zone. The selected material parameters were density $\rho=1.18 \mathrm{~g} / \mathrm{cm}^{3}$, Young's modulus $E=435 \mathrm{MPa}$, and shear modulus $G=159 \mathrm{MPa}$ for the epoxy background; $\rho=1.3 \mathrm{~g} / \mathrm{cm}^{3}, E=0.1175 \mathrm{MPa}$, and $G=0.04 \mathrm{MPa}$ for the silicone rubber; and $\rho=7.78 \mathrm{~g} / \mathrm{cm}^{3}, E=201.6 \mathrm{GPa}$, and $\mathrm{G}=81 \mathrm{GPa}$ for the steel square columns.

The Acoustic-solid Interaction (frequency domain) module was used to calculate the transmission spectrum. Finite stacked units are considered on the $z$ axis, and infinite units along $x$ and $y$ axes are considered using Bloch-Floquet periodic boundary conditions. It was assumed that a plane sound wave is incident along the $z$ axis and used transmission coefficients to represent the transmission spectrum of the stacked-structure LRSM. To reduce reflection at boundaries, perfectly matched layers were chosen to model infinite air domains at the top and bottom.

\section{Results and discussion}

\subsection{Band gap formation and coupling effect}

The stacked-structure LRSMs are transformed from two-dimensional LRSMs, and we thus discuss first the differences in their band structures. The band structures of the two-dimensional LRSM and stacked-structure LRSM are shown in Fig. 2(a) and (b), respectively. The width of the square rods is $0.3 \mathrm{~cm}$. The thickness of the coating materials is $0.1 \mathrm{~cm}$, and the lattice constant is $1 \mathrm{~cm}$. For the two-dimensional structure, the displacement can be described by two independent parts: the pure transverse displacement along the $z$ axis (dashed line in Fig. 2(a)) and the displacement of mixed longitudinal and transverse modes in the $x, y$ plane (solid line in Fig. 2(a)). The band gap is between 1703.4 and $2227.9 \mathrm{~Hz}$ for the modes in the $x, y$ plane, and between 605.4 and $797.93 \mathrm{~Hz}$ for modes out of the $x, y$ plane (as shown in Fig. 2(a)). Considering all types of vibration polarization (i.e., longitudinal and transverse), there are no absolute band gaps in the two-dimensional LRSM. For the stacked structure, there is a band gap between 1758.9 and $2411.5 \mathrm{~Hz}$. Owing to its three-dimensional structure, the band gap adapts to all types of vibration polarization (as shown in Fig. 2(b)). The bandwidth of the stacked-structure LRSM is approximately $652.6 \mathrm{~Hz}$, which is approximately $128 \mathrm{~Hz}$ broader than that of the two-dimensional LRSM in the $x, y$ plane. It is thus easier to realize a wider band gap that can be adapted to different polarization states with the stacked-structure LRSM.

The vibration modes of lower and upper edges of band gaps in the $x, y$ plane for the two-dimensional LRSM are shown in Fig. 3(a) and (b). It is seen that the vibration mode of the lower edge of the band gap is the vibration of a metal block considered as one particle, and a fixed delay in the phases of the vibrations between adjacent lattices maintains the dynamic balance of the whole system (Fig. 3(a)) [25,28]. For the lower edge of the two-dimensional LRSM, the vibration model can be simplified as a mass-spring system (as shown in Fig. 3(c)), in which particle $m$ represents the equivalent mass of the oscillator (steel block) and spring $k$ represents the equivalent stiffness (of the coating material) of the oscillator. Additionally, the frequency of the lower edge can be evaluated as [36]

$$
f_{1}=\frac{1}{2 \pi} \sqrt{\frac{k}{m}} .
$$



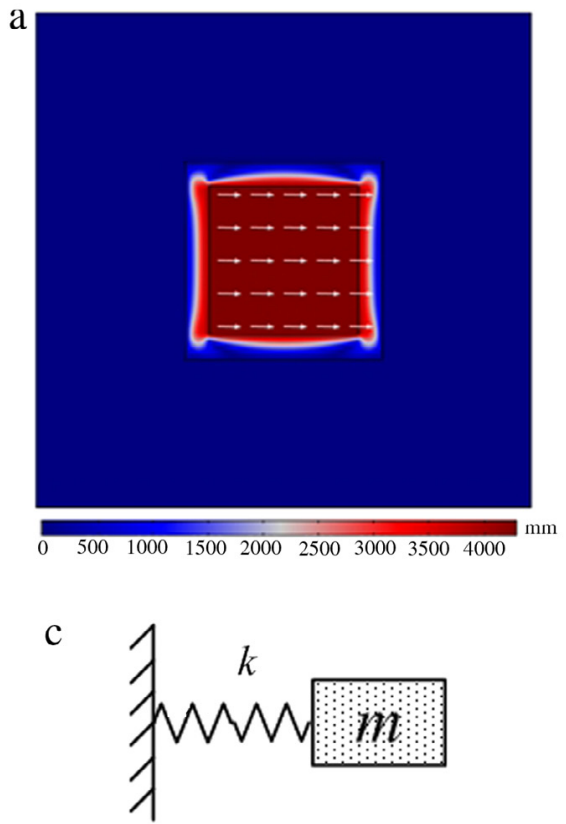

$\mathrm{b}$

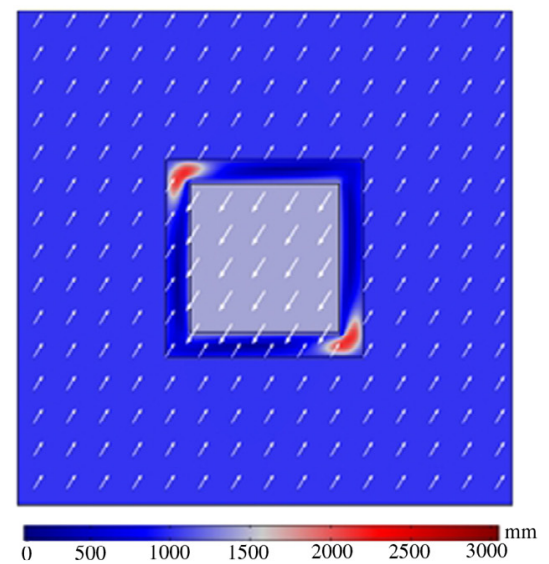

d

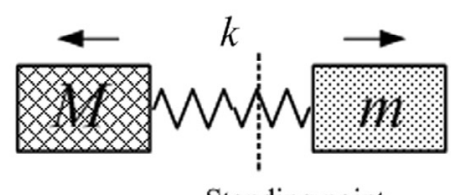

Standing point

Fig. 3. Vibration modes and mass-spring models of the two-dimensional LRSM in the $x, y$ plane: (a)(c) lower edge and (b)(d) upper edge of the band gap.

In contrast, the vibration mode of the upper-edge band gap is the vibration of the host media with the reversed phase of the metal block (as shown in Fig. 3(b)) [24,27]. For the upper edge of the two-dimensional LRSM, the vibration model can be simplified as a mass-spring-mass system (as shown in Fig. 4(b)), in which particles $m$ and $M$ respectively represent the equivalent mass of the steel block and matrix, and spring $k$ represents the equivalent stiffness of the coating material. The frequency of the lower edge can be evaluated as [36]

$$
f_{2}=\frac{1}{2 \pi} \sqrt{\frac{k(m+M)}{m M}} .
$$

The vibration modes at band gap edges out of the $x, y$ plane are similar to those in the $x, y$ plane, and their frequencies can also be evaluated with formulas (4) and (5). However, the value of the equivalent stiffness varies greatly between the two polarized modes. The compression or tension deformation produced by the tensile modulus of coating materials plays a dominant role in the $x, y$ plane, and $k$ is thus a function of tensile modulus $E$. Meanwhile, the shear deformation produced by the shear modulus dominates out of the $x, y$ plane, and $k$ is thus a function of shear modulus $G$. Because the tensile modulus is often greater than the shear modulus for common materials, frequencies of the band gap in the $x, y$ plane are usually higher than those of out of the $x, y$ plane, and it is difficult to overlap their band gaps in order to produce absolute band gaps for the two-dimensional LRSM. According to the vibration modes, the coupling between adjacent resonances is weak in the two-dimensional LRSM.

For the stacked-structure LRSM, the vibration mode at the lower edge is the vibration of one steel column along the $z$ axis, and the other columns are stationary relative to the matrix (Fig. 4(a)). We can make the same simplification of a mass-spring model in Fig. 4(c) as made for the vibration mode of the two-dimensional LRSM in the $x, y$ plane. The frequency is evaluated as

$$
f_{3}=\frac{1}{2 \pi} \sqrt{\frac{k_{E}}{m}},
$$

where $k_{E}$ is a function of tensile modulus $E$. The form of formula (6) is the same as that of formula (4), and the calculated frequency is similar to that of the lower edge of the two-dimensional LRSM.

The vibration mode of the upper-edge of the band gap (as shown in Fig. 3(b)) is one steel column perpendicular to the $y$ direction vibrating with the matrix and the adjacent column parallel to the $y$ direction being stationary. The resonances exist in one unit cell at the upper-edge of the band gap: compression behavior (compression resonance subject to the tensile modulus) and shear behavior (shear resonance subject to the shear modulus). The compression behavior is the relative vibration between the vibrating column and matrix, and is similar to vibration of the two-dimensional LRSM in the $x, y$ plane. Shear behavior is the vibration between the matrix and the stationary column, and is similar to vibration of the two-dimensional LRSM out of the $x, y$ plane. We can simplify the vibration mode of the upper-edge band gap as a mass-spring-mass-spring model (Fig. 4(d)). As shown in Fig. 4(d), although there are two masses connected in series for 
a

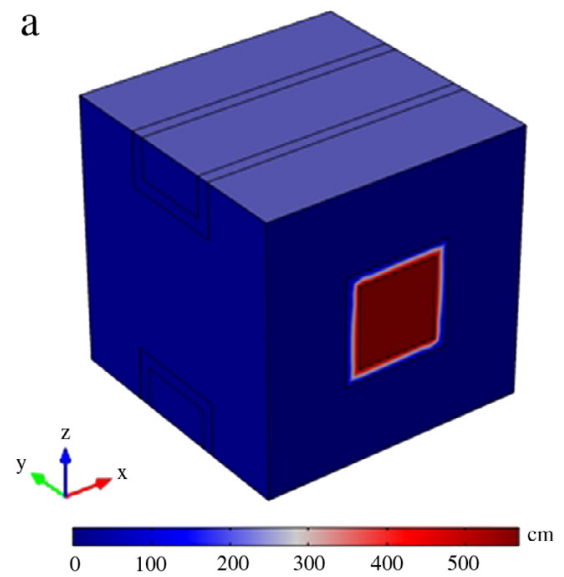

$\mathrm{C}$

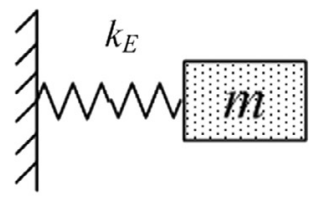

$\mathrm{b}$

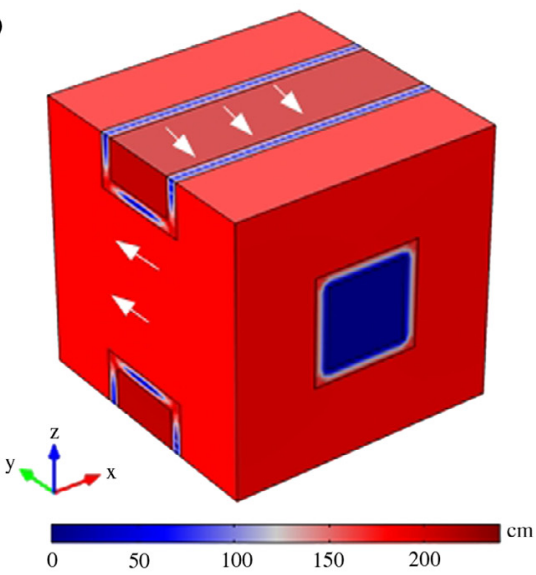

$\mathrm{d}$

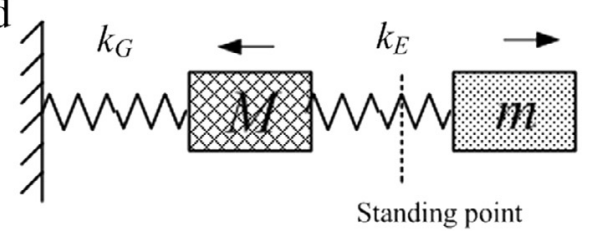

Fig. 4. Vibration modes and mass-spring models of the stacked-structure LRSM: (a)(c) lower edge and (b)(d) upper edge of the band gap.

the vibration mode of the upper-edge band gap, the masses have opposite vibratory direction, thus the resonant frequency is higher than the resonant frequency of the mass-spring model as shown in Fig. 4(c). We evaluate the upper-edge frequency as

$$
f_{4}=\frac{1}{2 \pi} \sqrt{\frac{\left(m k_{G}+M k_{E}+m k_{E}\right)+\sqrt{\left(m k_{G}+M k_{E}+m k_{E}\right)^{2}-4 m M k_{G} k_{E}}}{2 m M}},
$$

which are respectively functions of shear modulus $G$ and tensile modulus $E$. The equivalent stiffness $\left(k_{G}\right.$ and $\left.k_{E}\right)$ and mass $(M$ and $m$ ) can be obtained by the formula shown in Ref. [36]. Both formulas (6) and (7) contain $k_{E}$, and the value of $f_{4}$ is higher than that of $f_{3}$, thus opening a band gap. In addition, compared with formula (5), formula (7) includes the effect of sheardeformation-induced shear resonance between the matrix and stationary column. It is observed here that the frequency shifts to a higher value and the band gap widens. The above analysis reveals that there is strong coupling between cross resonances at the upper-edge band gap of the stacked-structure LRSM originating from the interaction between compression resonance and shear resonance. This strong coupling produces a band gap adaptable to all polarization states when the wave travels along the $z$ axis. In addition, both shear and compression deformations appear in coupled resonances, and the frequency is thus higher and the band gap wider. It is seen that the coupling effect is beneficial to the formation of a wider band gap.

For other types of LRSMs with complex lattices, such as multicoaxial cylindrical LRSMs [29], although multi-scale resonators have been introduced into one unit cell, the result has been multiple band gaps and the band gaps have not obviously widened. This is because the resonators with different frequencies vibrate in the same plane, and it is thus difficult to generate strong coupling between the resonators. In the stacked-structure LRSM, the resonators in one unit cell are intentionally arranged in perpendicular directions, and they have the same resonant frequencies. The orthogonal resonators act together at the same frequency, thus readily generating strong coupling. It is seen that the coupling strength is related to the arrangement of resonators in the unit cell.

The FEM was applied to investigate the transmission spectrum and better represent the band gap of the stacked-structure LRSM. A plane sound wave in an air background is incident on a stacked-structure LRSM having four units along the $z$ axis and an infinite number along $x$ and $y$ axes. By sweeping the frequency, the transmission coefficients of the stacked-structure LRSM in an air background were calculated as shown in Fig. 5. It is seen that there are two peaks at 1750 and $2850 \mathrm{~Hz}$, and a dip at $1850 \mathrm{~Hz}$. The dip results from the band gap in which wave propagation is inhibited. The location of the dip agrees with the band gap region $(1750-2400 \mathrm{~Hz})$. The peaks are the result of resonances in which displacement along the $z$ axis is dominant. The frequency of $1750 \mathrm{~Hz}$ is the lower band gap edge where the mass block vibration runs along the $z$ axis and has the reverse phase in the adjacent unit cell, and thus couples with the sound wave traveling along the $z$ axis to provide a magnified transmission coefficient. At $2850 \mathrm{~Hz}$, although the frequency is not the edge frequency of the band gap, the vibration mode is generated by the relative vibration of the matrix and steel column along the $z$ axis. The transmission spectrum suggests that the stacked-structure LRSM can be used as an acoustic filter or sound insulator. 


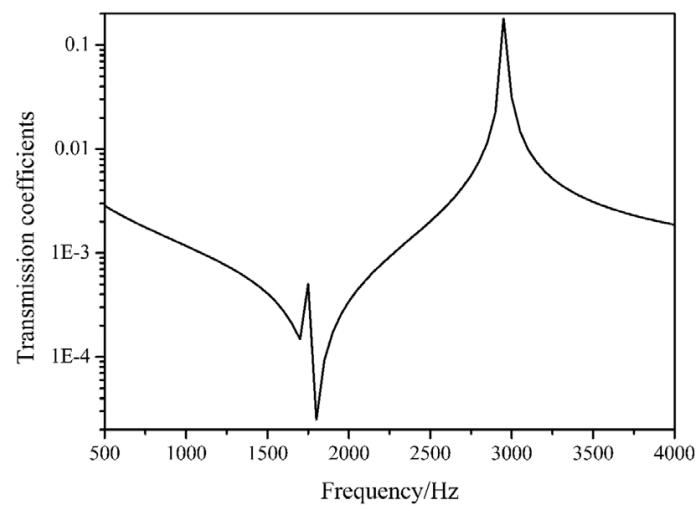

Fig. 5. Transmission coefficients of the stacked-structure LRSM in an air background.

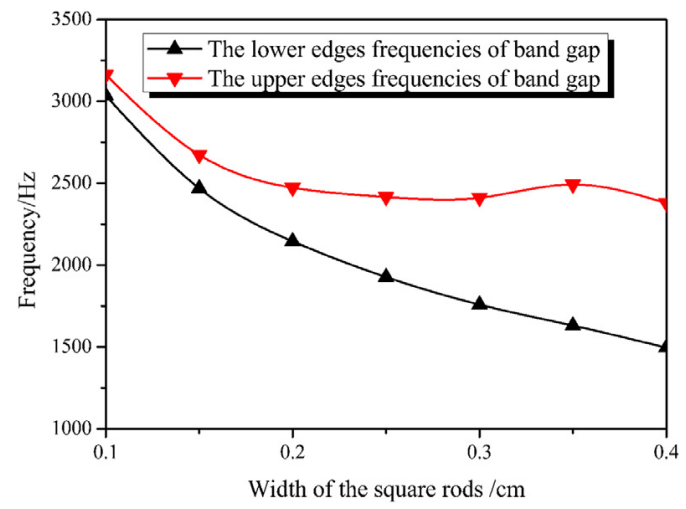

Fig. 6. Band gap edge frequencies of stacked-structure LRSMs having various widths of the square rod.

\subsection{Factors affecting the band gap and strength of the coupling effect}

As discussed above, the coupling between orthogonal resonances can open a wider band gap in a stacked-structure LRSM. The location of the band gap is determined by the vibrator (mass-spring system) and affected by the strength of the coupling. In the stacked-structure LRSM, the square rods act as the mass block and have a direct relationship with the frequencies of the band gap. To study how the width of the square rods affects the band gap and strength of the coupling, we kept the thickness of coating materials at $0.1 \mathrm{~cm}$ and the lattice constant at $1 \mathrm{~cm}$. Fig. 6 illustrates the dependency of the edge frequencies on the width of the square rods. It is seen that the lower-edge frequency depends on the scatter range in the stacked-structure LRMS, as for the two-dimensional LRSM owing to their similar vibration modes, and lower-edge frequencies of LRSMs both decrease as the width increases. The vibration mode of the lower band gap edge is the vibration of a metal block considered as a mass block (as shown in Fig. 4(a)). As the width increases, the oscillator mass increases, tending to shift the loweredge frequency toward lower values according to formula (6). Contrasting with the case for the two-dimensional LRSM, the upper-edge frequency first decreases and then increases (excluding the value for a rod width of $0.4 \mathrm{~cm}$ ) owing to the coupling effect. The sample having a square-rod width of $0.4 \mathrm{~cm}$ is an exceptional case discussed later. When the mass of the square rods is insufficient, the vibration mainly depends on one rod along the $y$ axis. The shear deformation around the stationary column is negligible, and the coupling effect is thus weak. Formula (7) can be approximated to formula (5), where the upper-edge frequency decreases with an increase in the rod width. As the width increases to a certain value, the matrix vibrates with the vibrating rod along the $y$ axis, and shear deformation around the stationary rod takes effect. Here, strong coupling prevails, and the upper-edge frequency increases with an increase in the square-rod width. In addition, it is found that the bandwidth broadening and coupling enhance with an increase in the square-rod width.

We also investigated the band gap of a stacked-structure LRSM consisting of steel rods of two widths $(0.3$ and $0.4 \mathrm{~cm})$ arranged in perpendicular directions. The calculation results are shown in Fig. 7. There is a band gap between 1757.3 and $2507.38 \mathrm{~Hz}$ along the $z$ axis. The band width is approximately $100 \mathrm{~Hz}$ broader than that for the stacked-structure LRSM having uniform rod width of $0.3 \mathrm{~cm}$ (as shown in Fig. 2(b)). The vibration mode at the lower-edge frequency is the same as that for the stacked-structure LRSM having uniform rod width of $0.3 \mathrm{~cm}$, as shown in Fig. 4(a), because it represents vibration of light rods (having width of $0.3 \mathrm{~cm}$ ) along the $z$ axis. The upper-edge vibration mode is generated by the relative vibration of the matrix and heavy rods (having width of $0.4 \mathrm{~cm}$ ) along the $y$ axis. Owing to the strong coupling, the upperedge frequencies increase with the width of the square rods (Fig. 6). Hence, the upper-edge frequency tends toward high 


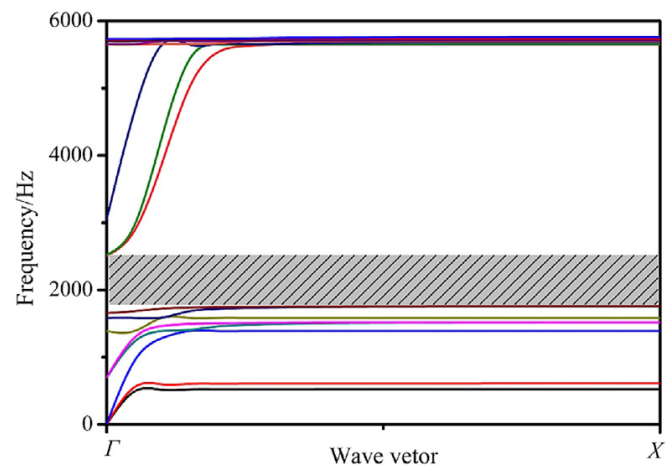

Fig. 7. Band structure of a stacked-structure LRSM with square-rod widths of 0.3 and $0.4 \mathrm{~cm}$.

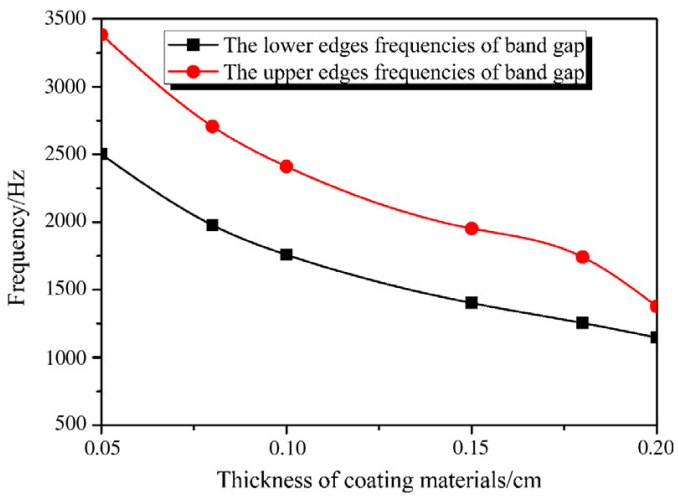

Fig. 8. Band gap edge frequencies of stacked-structure LRSMs with coating materials of varying thickness.

values for the stacked-structure LRSM consisting of rods with widths of 0.3 and $0.4 \mathrm{~cm}$. In addition, not all stacked-structure LRSMs consisting of differently sized steel rods have band gaps. For example, wood-pile LRSMs that have 0.3- and 0.1-cm or 0.4- and $0.1-\mathrm{cm}$ square rods have no band gaps. This is because the vibration frequency of light rods (mostly appearing at the lower edge of the band gap) along the $z$ axis is higher than the vibration frequency of heavy rods (mostly appearing at the upper edge of the band gap) along the $y$ axis.

In locally resonant materials, the coating layer serves as a spring, and its thickness affects the stiffness of the spring, making it critical to the band gap structure. To study how the coating material thickness affects the band gap and coupling strength, we kept the width of square rods at $0.3 \mathrm{~cm}$ and the lattice constant at $1 \mathrm{~cm}$. The calculation results are shown in Fig. 8, revealing that the trend of the change in the band gap with the thickness of the coating material in the stackedstructure LRSM is the same as the trend for most traditional LRSMs. Both the lower- and upper-edge band gap frequencies decrease as the thickness of the coating material increases, with slight bandwidth narrowing. According to the theory of locally resonant phononic crystal, when the thickness of the coating materials increases, the stiffness, $k$, decreases, reducing the band gap frequencies [36].

Moreover, it is noted that the band gaps of the samples with coated-rod widths of $0.5 \mathrm{~cm}$ (the case of $0.4-\mathrm{cm}$ square rods shown in Fig. 6 and that of 0.3-cm square rods shown in Fig. 8) are abnormal. In the present calculations, the lattice constant is kept at $1 \mathrm{~cm}$, and crossed coating materials thus come into contact with each other when the coated-rod width is $0.5 \mathrm{~cm}$. In this case, the dispersion curves are very different from those discussed above, and the bandwidth and upper-edge band frequency are dramatically less for the 0.5 - $\mathrm{cm}$ coated rods. Fig. 9(a) and (b) show the vibration modes of samples with 0.5$\mathrm{cm}$ coated rods and 0.4-cm square rods at the edge band frequencies. These figures present vibration modes different from those discussed above. The vibration mode of the lower edge of the band gap is the vibration of a metal block considered as a mass block (as shown in Fig. 9(a)) along the $z$ axis. Unlike earlier samples, both crossed rods vibrate with the coating materials, and their vibrations are reverse in phase to keep the unit balance. The vibration mode of the upper edge of the band gap is the vibration of the matrix considered as a mass block (as shown in Fig. 9(b)) along the $z$ axis, and the crossed rods are stationary. Compared with the stacked-structure LRSM with coating materials not in contact, both the upper-and lower-edge band frequencies tend toward lower values, and the coupling disappears.

In addition, the viscoelastic loss of each material was considered to investigate the damping influence on the band gap of the stacked-structure LRSM. For ternary LRSMs, matrix and coating materials are usually polymers which inevitably 
a

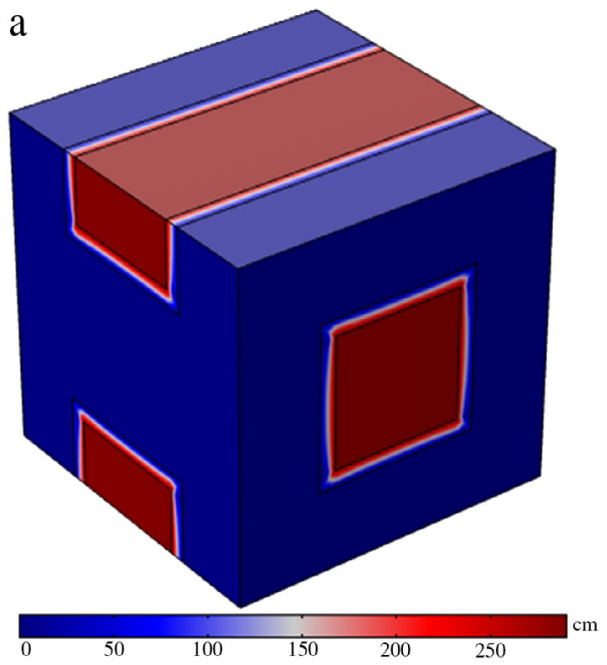

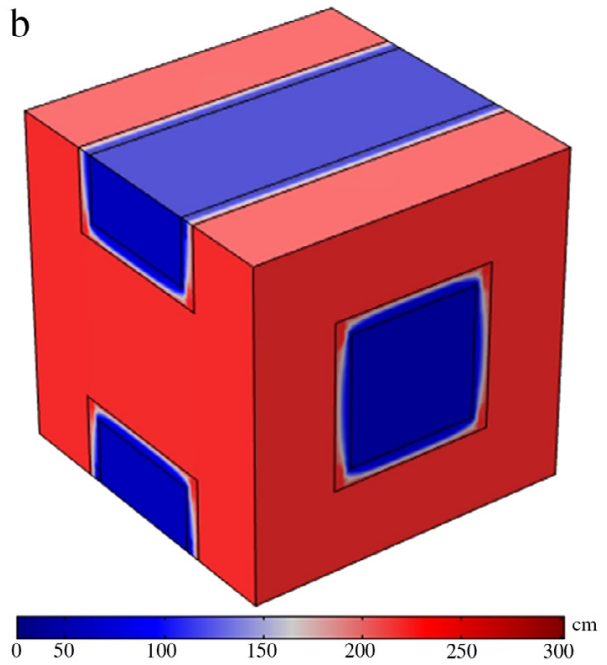

Fig. 9. Vibration modes of the lower edge (a) and upper edge (b) of the band gap of the stacked-structure LRSM with a square-rod width of $0.4 \mathrm{~cm}$, coating material thickness of $0.1 \mathrm{~cm}$, and lattice constant of $1 \mathrm{~cm}$
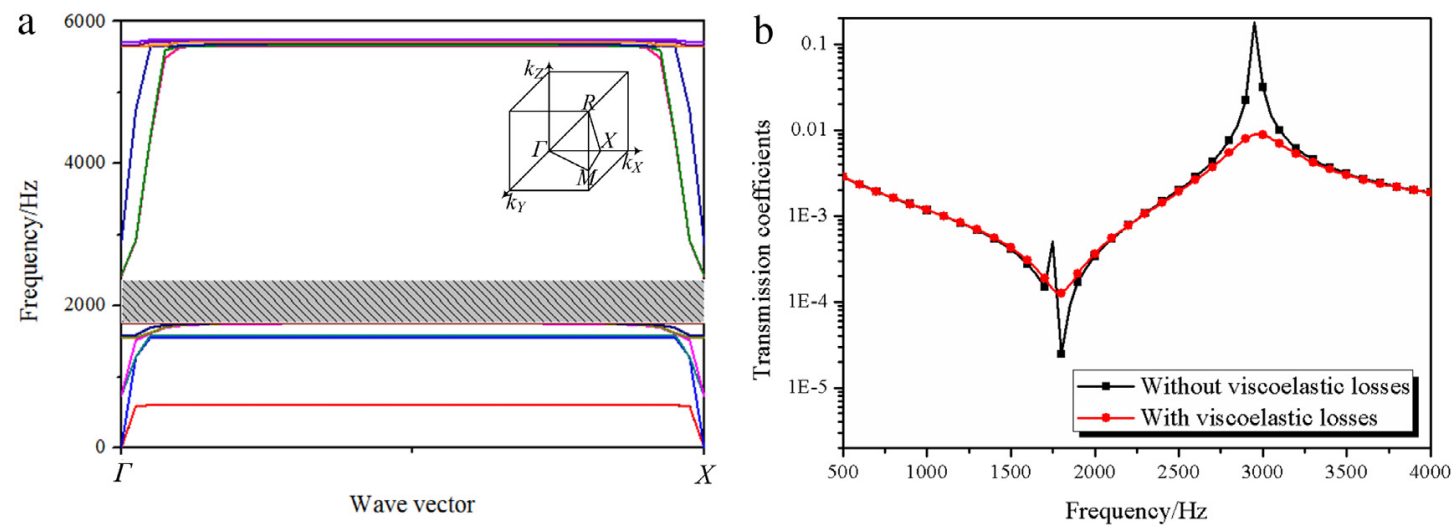

Fig. 10. Band structure (a) and transmission coefficients (b) of stacked-structure LRSM with viscoelastic losses; square-rod width 0.3 cm, coating material thickness $0.1 \mathrm{~cm}$, lattice constant $1 \mathrm{~cm}$.

contain viscoelasticity. It was found that the presence of viscoelastic damping enhanced the sound absorption at resonant frequencies [32,33], and likely also influenced the displacement and width of the band gap of the LRSMs [37-39]. In this study, the matrix and coating materials of the stacked-structure LRSMs were, respectively, pure epoxy and silicone rubber, the damping losses of which are very low. When examining the damping's influence on the band gap of the stacked-structure LRSM, isotropical loss factors were used to represent the viscoelasticity of materials and the moduli were kept unchanged, as mentioned above. The loss factors of the pure epoxy and silicone rubber at room temperature are 0.05 and 0.1 [40], respectively. Calculations are shown in Fig. 10.

The band structures and transmission coefficients of the stacked-structure LRSM with viscoelastic losses are shown in Fig. 10. When viscoelasticity was accounted for, there was a band gap between 1761.1 and $2414.5 \mathrm{~Hz}$ (Fig. 10(a)). Compared to the calculations without viscoelastic losses (Fig. 2(b)), both the lower- and upper-edge of the band gap shifted slightly to higher frequency (about $3 \mathrm{~Hz}$,) and the bandwidth was nearly invariable. In effect, there are no obvious differences between the two cases-viscoelastic losses have negligible impact on the band structure of the stacked-structure LRSM. When considering the damping losses, the transmission coefficients were as shown in Fig. 10(b), where the location of the dip matched the band gap region. Compared to the calculations without viscoelastic losses (Fig. 5), the sharp peaks and dips in the resonant states were washed out due to the dissipation of the matrix and coating materials [37]. These results show that viscoelastic damping mainly influences transmission, and has little effect on the band structure.

The above calculations suggest the band gap structure and coupling strength are affected by the width and size of the square rods and the thickness and manner of contact of the coating material. With this knowledge, the band gap of the stacked structure can be tuned readily by changing various structural factors. In addition, the width of the band gap was found to have a close relationship with the strength of coupling between the resonances. Strong coupling broadens the band gap more effectively. 


\section{Conclusion}

This study systematically investigated the band structures and vibration modes of stacked-structure LRSMs to study coupling between resonances. The observed coupling produces a wider band gap that can be adapted to all types of vibration polarization. The calculated vibration modes and mass-spring model verify that strong coupling exists between orthogonal resonances (the shear resonance and compression resonance) at the upper edge of the band gap, and provides a wider bandwidth. In addition, how the width of the square rods and thickness of the coating material affect the band gap and coupling strength was investigated. It was found that the bandwidth has a close relationship with the strength of coupling between resonations. The results suggest a novel design for broad band gaps, leading to further opportunities for practical applications of LRSM.

\section{Acknowledgments}

We acknowledge project supported by the National Natural Science Foundation of China (Grant Nos. 11202211 and 11272315) and the opening project of State Key Laboratory of Explosion Science and Technology (Beijing Institute of Technology). The opening project number is KFJJ14-5M.

\section{References}

[1] Z. Liu, X. Zhang, Y. Mao, Y. Zhu, Z. Yang, C.T. Chan, P. Sheng, Locally resonant sonic materials, Science 289 (2000) 1734-1736.

[2] Y. Lai, Y. Wu, P. Sheng, Z.Q. Zhang, Hybrid elastic solids, Nature Mater. 10 (2011) 620-624.

[3] N. Fang, D. Xi, J. Xu, M. Ambati, W. Srituravanich, C. Sun, X. Zhang, Ultrasonic metamaterials with negative modulus, Nature Mater. 5 (2006) $452-456$.

[4] W.J. Parnell, T. Shearer, Antiplane elastic wave cloaking using metamaterials, homogenization and hyperelasticity, Wave Motion 50 (2013) $1140-1152$.

[5] A.S. Titovich, A.N. Norris, Tunable cylindrical shell as an element in acoustic metamaterial, J. Acoust. Soc. Am. 136 (2014) 1601-1609.

[6] K.T. Tan, H.H. Huang, C.T. Sun, Blast-wave impact mitigation using negative effective mass density concept of elastic metamaterials, Int. J. Impact Eng. 64 (2014) 20-29.

[7] E. Kim, J. Yang, Wave propagation in single column woodpile phononic crystals: Formation of tunable band gaps, J. Mech. Phys. Solids 71 (2014) $33-45$.

[8] E. Kim, F. Li, C. Chong, G. Theocharis, J. Yang, P.G. Kevrekidis, Highly nonlinear wave propagation in elastic woodpile periodic structures, Phys. Rev. Lett. 114 (2015) 118002. 1-5.

[9] Z. Liu, C.T. Chan, P. Sheng, Three-component elastic wave band-gap material, Phys. Rev. B 65 (2002) 165116.

[10] P. Sheng, X. Zhang, Z. Liu, C. Chan, Locally resonant sonic materials, Physica B 338 (2003) 201-205.

[11] M. Hirsekorn, Small-size sonic crystals with strong attenuation bands in the audible frequency range, Appl. Phys. Lett. 84 (2004) $3364-3366$.

[12] H. Zhao, Y. Liu, J. Wen, D. Yu, X. Wen, Tri-component phononic crystals for underwater anechoic coatings, Phys. Lett. A 367 (2007) $224-232$.

[13] M.I. Hussein, M.J. Leamy, M. Ruzzene, Dynamics of phononic materials and structures: Historical origins, recent progress, and future outlook, Appl. Mech. Rev. 66 (2014) 040802.

[14] K.M. Ho, C.K. Cheng, Z. Yang, X. Zhang, P. Sheng, Broadband locally resonant sonic shields, Appl. Phys. Lett. 83 (2003) 5566-5568.

[15] X. Zhang, Y. Liu, F. Wu, Z. Liu, Large two-dimensional band gaps in three-component phononic crystals, Phys. Lett. A 317 (2003) 144-149.

[16] Y. Gu, X. Luo, H. Ma, Optimization of the local resonant sonic material by tuning the shape of the resonator, J. Phys. D: Appl. Phys. 41 (2008) 205402.

[17] Y. Gu, X. Luo, H. Ma, Low frequency elastic wave propagation in two dimensional locally resonant phononic crystal with asymmetric resonator, J. Appl. Phys. 105 (2009) 044903.

[18] Y.F. Wang, Y.S. Wang, L. Wang, Two-dimensional ternary locally resonant phononic crystals with a comblike coating, J. Phys. D: Appl. Phys. 47 (2014) $015502-015509$

[19] Z. Liu, S. Yang, X. Zhao, Chin. ultrawide bandgap locally resonant sonic materials, Phys. Lett. 22 (2005) 3107-3110.

[20] B.S. Lazarov, J.S. Jensen, Low-frequency band gaps in chains with attached non-linear oscillators, Int. J. Non-Linear Mech. 42 (2007) 1186-1193.

[21] X. Zhou, C. Chen, Tuning the locally resonant phononic band structures of two-dimensional periodic electroactive composites, Physica B 431 (2013) $23-31$.

[22] H. Meng, J. Wen, H. Zhao, X. Wen, Optimization of locally resonant acoustic metamaterials on underwater sound absorption characteristics, J. Sound Vib. 331 (2012) 4406-4416.

[23] Y. Xiao, B.R. Mace, J. Wen, X. Wen, Formation and coupling of band gaps in a locally resonant elastic system comprising a string with attached resonators, Phys. Lett. A 375 (2011) 1485-1491.

[24] M.B. Assouar, M. Oudich, Dispersion curves of surface acoustic waves in a two-dimensional phononic crystal, Appl. Phys. Lett. 99 (2011) 123505.

[25] M.B. Assouar, J. Sun, F. Lin, J. Hsu, Hybrid phononic crystal plates for lowering and widening acoustic band gaps, Ultrasonics 54 (2014) $2159-2164$.

[26] Y. Cheng, J. Xu, X. Liu, Broad forbidden bands in parallel-coupled locally resonant ultrasonic metamaterials, Appl. Phys. Lett. 92 (2008) 051913.

[27] A.-C. Hladky-Hennion, G. Allan, M.D. Billy, Localized modes in a one-dimensional diatomic chain of coupled spheres, J. Appl. Phys. 98 (2005) 054909.

[28] H. Zhao, Y. Liu, G. Wang, J. Wen, D. Yu, X. Han, X. Wen, Resonance modes and gap formation in a two-dimensional solid phononic crystal, Phys. Rev. B $72(2005) 012301$.

[29] H. Larabi, Y. Pennec, B. Djafari-Rouhani, J.O. Vasseur, Multicoaxial cylindrical inclusions in locally resonant phononic crystals, Phys. Rev. E 75 (2007) 066601.

[30] M. Hirsekorn, P.P. Delsanto, A.C. Leung, P. Matic, Elastic wave propagation in locally resonant sonic material: Comparison between local interaction simulation approach and modal analysis, J. Appl. Phys. 99 (2006) 124912.

[31] Z. Liu, C.T. Chan, P. Sheng, Analytic model of phononic crystals with local resonances, Phys. Rev. B 71 (2005) 014103.

[32] H. Jiang, Y. Wang, M. Zhang, Y. Hu, D. Lan, Y. Zhang, B. Wei, Locally resonant phononic woodpile: A wide band anomalous underwater acoustic absorbing material, Appl. Phys. Lett. 95 (2009) 104101.

[33] J. Wen, H. Zhao, L. Lv, B. Yuan, G. Wang, X. Wen, Effects of locally resonant modes on underwater sound absorption in viscoelastic materials, J. Acoust. Soc. Am. 130 (2011) 1201-1208.

[34] I.A. Veres, T. Berer, O. Matsuda, Complex band structures of two dimensional phononic crystals: Analysis by the finite element method, J. Appl. Phys. 114 (2013) 083519

[35] M. Oudich, Y. Li, B.M. Assouar, Z. Hou, A sonic band gap based on the locally resonant phononic plates with stubs, New J. Phys. 12 (2010) 083049.

[36] G. Wang, L. Shao, Y. Liu, J. Wen, Accurate evaluation of lowest band gaps in ternary locally resonant phononic crystals, Chin. Phys. 15 (2006) $1843-1848$.

[37] I.E. Psarobas, Viscoelastic response of sonic band-gap materials, Phys. Rev. B 64 (2001) 012303.

[38] Y. Liu, D. Yu, H. Zhao, J. Wen, X. Wen, Theoretical study of two-dimensional phononic crystals with viscoelasticity based on fractional derivative models, J. Phys. D 41 (2008) 065503.

[39] R.P. Moiseyenko, V. Laude, Material loss influence on the complex band structure and group velocity in phononic crystals, Phys. Rev. B 83 (2011) 064301.

[40] Q. Sun, H. Wang, P. Zhang, X. Cheng, Z. Fan, Development of damping silicone rubber, Spec. Purp. Rubber Prod. 27 (2006) 58-62. 